\title{
Overlapping presentation of fungal tubulointerstitial nephritis in an immunosuppressed pediatric patient
}

\author{
James B. Tee • Vivian Reznik • Henry F. Krous
}

Received: 6 October 2008 /Revised: 4 February 2009 / Accepted: 18 February 2009/Published online: 19 March 2009

(C) The Author(s) 2009. This article is published with open access at Springerlink.com

\begin{abstract}
With the expanding use of immunosuppressive therapies and broad-spectrum antibiotics, Candida species has become an increasingly important cause of infections, particularly in the presence of anti-tumor necrosis factor- $\alpha$ therapy. We report the case of a 17-year-old female with ulcerative colitis who developed oliguric renal failure following immunosuppressive and nephrotoxic therapy. Although urine cultures and urinary tract imaging were negative in the face of fungemia, renal biopsy was the key to establishing the diagnosis of fungal tubulo-interstitial nephritis as the primary reversible cause of the renal failure.
\end{abstract}

Keywords Antibodies · Case reports - Immunosuppression · Interstitial $\cdot$ Nephritis $\cdot$ Monoclonal $\cdot$ Mycoses $\cdot$ Pediatrics Tumor necrosis factor-alpha

\section{J. B. Tee}

Division of Pediatric Nephrology, Department of Pediatrics,

University of Calgary,

Calgary, AB, Canada

\section{Reznik}

Division of Pediatric Nephrology, Department of Pediatrics, University of California San Diego,

San Diego, CA, USA

\section{H. F. Krous}

Department of Pathology, Rady Children's Hospital of San Diego and University of California San Diego School of Medicine, San Diego, CA, USA

\section{J. B. Tee $(\square)$}

Alberta Children's Hospital,

2888 Shaganappi Trail NW,

Calgary, AB, Canada T3B 6A8

e-mail: james.tee@alumni.utoronto.ca

\section{Introduction}

The use of stronger biological agents for treating immunemediated diseases carries an increased risk of invasive opportunistic infections. With the expanding use of immunosuppressives and broad-spectrum antibiotics, fungi have become an increasingly important cause of infections. Candida species may have a predilection for renal involvement, as up to $90 \%$ of patients with disseminated candidiasis can have renal involvement at autopsy [1]. Acute renal failure in this population has been largely reported as a complication of urinary tract obstruction from fungal bezoar [2, 3]. Only a single case of acute tubulointerstitial nephritis from Candida albicans in a patient receiving steroids and antibiotics has been reported to date [4], but the concurrent use of nephrotoxic and immunosuppressive agents can mask the primary cause of acute renal failure. We report here for the first time a pediatric case of oliguric acute renal failure in an immunosuppressed patient with disseminated Candida species where renal biopsy led to the diagnosis of fungal tubulointerstitial nephritis in light of negative urine cultures and concurrent nephrotoxic therapy.

\section{Case report}

A 17-year-old female with ulcerative colitis presented with colonic inflammation on biopsy despite corticosteroid treatment. Blood and urine cultures were negative, and renal function was normal (serum creatinine $0.5 \mathrm{mg} / \mathrm{dL}$ ). Infliximab was initiated at a starting infusion dose of $5 \mathrm{mg} / \mathrm{kg}$, and the patient developed a fever. Laboratory test results were significant for an elevated white blood cell count $\left(29.3 \times 10^{3} / \mu \mathrm{L}\right)$ with band count $(82 \%)$ in the face of 
decreased renal function (serum creatinine $1.3 \mathrm{mg} / \mathrm{dL}$ ). Urinalysis revealed a specific gravity of 1.010, trace blood, and no protein, leukocytes, nitrites, casts, or other sediment. A blood culture was positive for Candida albicans. Neither a blood-to-urine $\beta 2$-microglobulin level nor plasma $\beta$-Dglucan level were available. Stool, cerebrospinal fluid, and urine cultures were negative. Drug therapy with intravenous fluconazole was initiated and then subsequently switched to caspofungin. A Doppler renal ultrasound revealed enlarged echogenic kidneys with no evidence of abscess or hydronephrosis. Magnetic resonance imaging confirmed the absence of systemic infiltrates, and an echocardiogram was normal. Repeat urine cultures remained negative. Renal function continued to decline with the emergence of oliguria (serum creatinine $7.1 \mathrm{mg} / \mathrm{dL}$ ). A percutaneous renal biopsy was performed, and hemodialysis was initiated for fluid overload and hyperphosphatemia.

The renal biopsy showed severe active tubulointerstitial nephritis with budding yeast forms and pseudohyphae consistent with Candida that were visualized within the tubules (Fig. 1). The interstitium and tubules were infiltrated with numerous neutrophils but not eosinophils. The tubular basement membranes were disrupted, indicating likely evolution to focal renal scarring. The glomeruli were essentially normal. Neither vasculitis nor granulomas were detected. Immunofluorescence microscopy was negative for immunoglobulins, complement, and fibrin.

Antifungal therapy was strengthened with the addition of liposomal amphotericin B administered both intravenously and via bladder irrigation. A urine culture performed after the renal biopsy returned positive for Candida albicans several

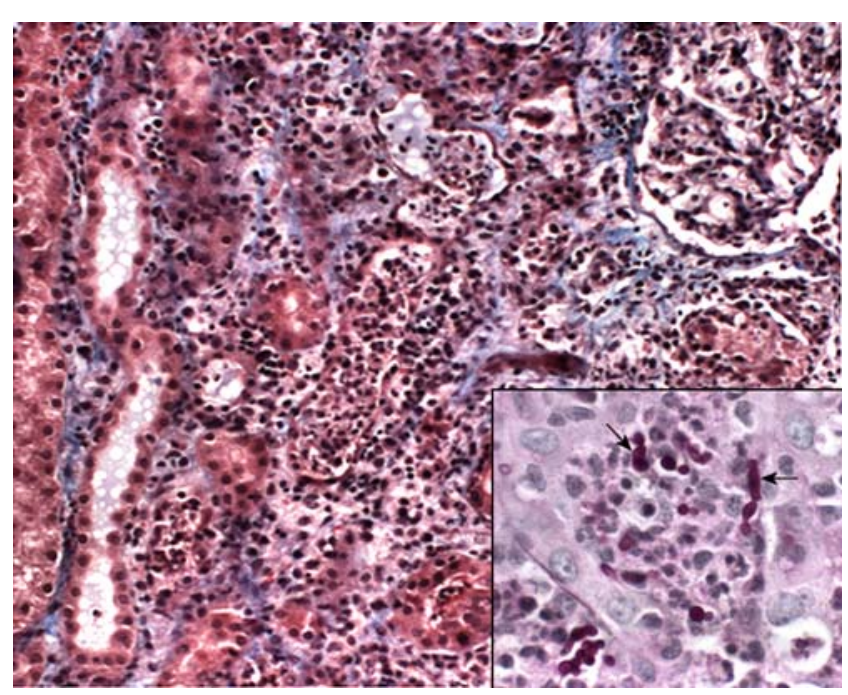

Fig. 1 Masson's trichrome staining of the kidney reveals severe tubulointerstitial disease and a relatively normal glomerulus. Magnification $\times 100$. Insert Fungal organisms (arrows) and neutrophils are present in the tubules and adjacent interstitium. Periodic acid-Schiff stain, magnification $\times 200$ days later. Within 1 year of discharge, the patient's serum creatinine had stabilized to a new baseline of $0.9 \mathrm{mg} / \mathrm{dL}$.

\section{Discussion}

Before the renal biopsy, the differential diagnosis for acute renal failure in this patient included medication-mediated nephrotoxicity, an autoimmune-mediated lupus-like syndrome or cortical necrosis that has been reported with infliximab, fungal nephritis (obstructive versus interstitial), and endocarditis-associated nephritis $[5,6]$. The absence of clinical signs of lupus together with a negative anti-double stranded DNA antibody made the classic diagnosis of infliximab drug-induced lupus unlikely [7]. The absence of vegetations on the echocardiogram as well as the absence of immune-complex-mediated staining on immunohistopathology ruled out endocarditis-associated nephritis. Doppler and magnetic resonance imaging highlighted the absence of an obstructing fungal bezoar of the urinary tract, which is the more typical presentation of fungal-associated acute renal failure [2, 3, 8].

As illustrated in this case, the overlapping use of antitumor necrosis factor (TNF) immunosuppressive therapy and nephrotoxic agents can complicate the diagnosis of acute renal failure. The use of infliximab, similar to the use of other anti-TNF- $\alpha$ antibody agents, presents a particularly strong risk for severe fungal infection via its binding to TNF, which is released by the immune system, constraining the patient's ability to respond to infectious stimuli $[9,10]$. The U.S. Food and Drug Administration (FDA) recently warned of an increase in reported invasive fungal infections with the use of TNF blockers, based on primarily adult case reports of pulmonary involvement in histoplasmosisendemic regions [11]. Percutaneous renal biopsy was key to the early establishment of fungal interstitial nephritis as a contributor to our patient's persistent renal failure, as standard urine cultures for fungi may take up to 4 weeks to become positive [12]. The diffuse visible yeast forms and pseudohyphae visualized within the tubules and interstitium on biopsy were striking given the prior absence of positive urinary cultures. Confirmation of a fungal cause for the renal failure allowed for targeted augmentation of antifungal therapy as well as further reductions in immunosuppressive therapy. This early detection and treatment of the fungal infection in the face of TNF-blockade with significant improvement in renal function on follow-up was consistent with other adult case studies and is supported by a recent FDA report that over half of the patients who experienced a delay in diagnosis ultimately died [4, 11].

In summary, the differential for acute renal failure in the immunosuppressed fungemic pediatric patient should include fungal tubulointerstitial nephritis, particularly when 
other fungal risk factors exist, such as with concurrent antiTNF- $\alpha$ therapy. Early detection of such invasive infections are of increasing importance with the expanded use of TNF-blockers to the pediatric patient population, and renal biopsy should be considered when clinical, laboratory and radiologic methods are inconclusive.

Funding for research support None.

IRB approval granted April 17th, 2008 (Rady Children's Hospital of San Diego, study reference no. 08021C).

Open Access This article is distributed under the terms of the Creative Commons Attribution Noncommercial License which permits any noncommercial use, distribution, and reproduction in any medium, provided the original author(s) and source are credited.

\section{References}

1. Lehner T (1964) Systemic candidiasis and renal involvement. Lancet 41:1414-1416

2. Babut JM, Coeurdacier P, Bawab F, Treguier C, Fremond B (1995) Urinary fungal bezoars in children-report of two cases. Eur J Pediatr Surg 5:248-252

3. Kale H, Narlawar RS, Rathod K (1985) Renal fungal ball: an unusual sonographic finding. J Clin Ultrasound 30:178-180
4. Ramsay AG, Olesnicky L, Pirani CL (1985) Acute tubulointerstitial nephritis from candida albicans with oliguric renal failure. Clin Nephrol 24:310-314

5. Stokes MB, Foster K, Markowitz GS, Ebrahimi F, Hines W, Kaufman D, Moore B, Wolde D, D'Agati VD (2005) Development of glomerulonephritis during anti-TNF-alpha therapy for rheumatoid arthritis. Nephrol Dial Transplant 20:1400-1406

6. Ahmed MM, Mubashir E, Dossabhoy NR (2007) Isolated renal sarcoidosis: a rare presentation of a rare disease treated with infliximab. Clin Rheumatol 26:1346-1349

7. De Rycke L, Kruithof E, Van Damme N, Hoffman IE, Van den Bossche N, Van den Bosch F, Veys EM, De Keyser F (2003) Antinuclear antibodies following infliximab treatment in patients with rheumatoid arthritis or spondylarthropathy. Arthritis Rheum 48:1015-1023

8. Fisher JF, Chew WH, Shadomy S, Duma RJ, Mayhall CG, House WC (1982) Urinary tract infections due to Canadia albicans. Rev Infect Dis 4:1107-1118

9. Crum NF, Lederman ER, Wallace MR (2005) Infections associated with tumor necrosis factor-alpha antagonists. Medicine (Baltimore) 84:291-302

10. Hage CA, Wood KL, Winer-Muram HT, Wilson SJ, Sarosi G, Knox KS (2003) Pulmonary cryptococcosis after initiation of antitumor necrosis factor-alpha therapy. Chest 124:2395-2397

11. U.S. Food and Drug Administration (FDA) (2008) Press release 4 September 2008. FDA, Washington D.C. Available at: http:// www.fda.gov/bbs/topics/NEWS/2008/NEW01879.html

12. Labarca JA, Wagar EA, Grasmick AE, Kokkinos HM, Bruckner DA (1998) Critical evaluation of 4-week incubation for fungal cultures: is the fourth week useful? J Clin Microbiol 36:36833685 